\title{
WHO Analgesic Method
}

National Cancer Institute

\section{Source}

National Cancer Institute. WHO Analgesic Method. NCI Thesaurus. Code C21122.

Developed to promote the sequential use of drugs to achieve effective pain relief. The first step is a non-opioid medication (such as aspirin, paracetamol, or ibuprofen). If this does not relieve the pain, an opioid for mild to moderate pain (such as codeine) should be added. When an opioid for mild to moderate pain in combination with a non-opioid medication does not provide effective analgesia, then an opioid for moderate to severe pain (such as morphine or one in the therapeutic group of morphine) should be substituted. Adjuvant drugs should be given at any point during drug treatment to relieve adverse effects of analgesics, to enhance pain relief, and to treat concomitant psychological disturbances such as insomnia, anxiety, and depression. (WHO) 